\title{
Acute dysfunction of Starr-Edwards mitral prostheses
}

\author{
JOHN GUNSTENSEN
}

\author{
Department of Thoracic Surgery, Royal Infirmary, Edinburgh
}

\begin{abstract}
Four cases of acute dysfunction of Starr-Edwards mitral prostheses are recorded. The patients presented with sudden dyspnoea 4 to 18 months after apparently successful mitral valve replacement. The prosthetic valve dysfunction was caused by thrombus on the bare metal cage of the prosthesis. No warning thromboembolic phenomena had been recorded. Urgent replacement of the valve resulted in the survival of one patient.
\end{abstract}

The replacement of diseased heart valves by various types of prosthetic valve is now an established procedure and the most popular valve is the Starr-Edwards prosthesis first used in 1960 (Starr and Edwards, 1961). Thromboembolism is a common occurrence in patients with prosthetic heart valves, but rarely does it produce acute dysfunction of the prosthesis months after insertion of the valve, a complication first recorded in 1964 (Garamella, Lynch, Schmidt, and Jensen, 1964).

In the five-year period from September 1965 to October 1970, 192 Starr-Edwards mitral prostheses were inserted at the Cardio-thoracic Unit in Edinburgh. Of these, 131 were ball valves ( 82 models 6000 and 6120 with bare metal struts and a silastic rubber ball, and 49 models 6300 and 6310 with cloth-covered struts and a metal ball) and one of these obstructed due to thrombus on the cage 18 months after insertion of the valve. Sixty-one of the Starr-Edwards valves inserted were low-profile disc valves (model 6500 with bare metal struts and a metal disc) and three of these failed acutely 4 to 15 months after apparently successful insertion. During this period the overall thromboembolic rate for Starr-Edwards mitral valve replacement was about $25 \%$.

The purpose of this paper is to record the presentation, diagnosis, and management of these four cases of acute dysfunction of Starr-Edwards mitral prostheses.

\section{CASE REPORTS}

CASE 1 A 51-year-old housewife, having had two previous closed mitral valvotomies, had her mitral valve replaced with a Starr-Edwards mitral ball prosthesis (model 6000) in October 1967. She made an uneventful recovery from operation, and anticoagulation with warfarin was started on the second postoperative day. At review in March 1969 she was well with normal valve and heart sounds, but in May 1969 (18 months after insertion of the prosthesis) several attacks of acute nocturnal dyspnoea occurred and two weeks later the patient was admitted to hospital in congestive cardiac failure with severe pulmonary oedema. She was breathless and cyanosed. The cardiac rhythm was that of atrial fibrillation with a pulse rate of $120 /$ $\mathrm{min}$ and the blood pressure was $100 / 60 \mathrm{mmHg}$. The neck veins were distended and there were numerous crepitations in both lungs. On auscultation of the heart the most striking finding was that the previously loud valve clicks were muffled and difficult to hear. A variable systolic murmur, conducted to the axilla, was heard at the apex. A diagnosis of acute prosthetic valve obstruction by thrombus was made. The patient improved greatly as a result of additional diuretic therapy and it was thought reasonable to delay operation for 48 hours to allow reversal of anticoagulation with vitamin K1. However, 24 hours after being given the vitamin $\mathrm{K} 1$ she suddenly deteriorated and operation was carried out forthwith. Residual anticoagulant activity was reversed with plasma prothrombin complex concentrate given intravenously.

A bilateral transverse thoracotomy was made, but before the pleural spaces had been opened asystole occurred. The heart was made to beat again with the intracardiac injection of adrenaline and the heart was then mobilized with considerable difficulty through thick adhesions from the adherent pericardium. Right atrial to ascending aorta cardiopulmonary bypass was established and the left atrium was opened. The atrial aspect of the prosthesis looked normal, but after excision of the prosthesis it was seen that a mass of clot adherent to one of the struts of the cage held the ball in its seating and almost entirely prevented ball movement (Fig. 1). A new Starr-Edwards prosthesis (model 6300) was inserted. The patient did not regain consciousness after operation and died 36 hours later.

At necropsy the right coronary artery was found to be occluded by thrombus some $3 \mathrm{~cm}$ from its origin and there was a posterior myocardial infarct. It was assumed that the period of cardiac arrest at the begin- 


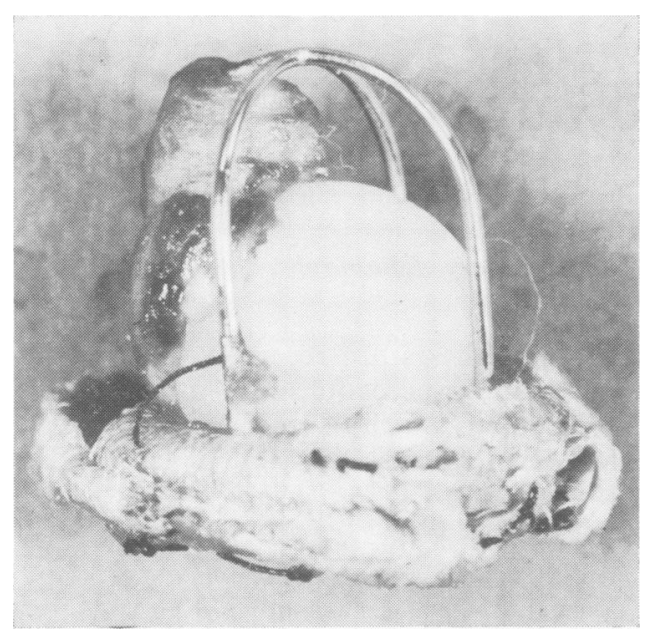

FIG. 1. Starr-Edwards ball valve prosthesis (model 6000) removed from case 1 . The thrombus on one of the struts of the cage is pushing the ball firmly into the seating.

ning of operation was responsible for the cerebral damage and that death was due to this cerebral damage and coronary embolism.

CASE 2 A 46-year-old housewife with severe rheumatic mitral disease had a closed mitral valvotomy in 1954 and again in 1957. In April 1969 a heavily sclerosed, but not calcified, mitral valve was replaced with a Starr-Edwards low-profile disc prosthesis (model 6500). The operation and postoperative recovery were uneventful. Anticoagulation was started on the second postoperative day using warfarin. The patient remained well for four months, but in August 1969 she suddenly experienced an attack of nocturnal dyspnoea when at home and 48 hours later she was admitted to hospital in a moribund state. The clicks of the prosthesis were not audible. She was transferred at once to the operating theatre and anticoagulation was reversed with plasma prothrombin complex concentrate given intravenously.

Before opening the chest partial cardiopulmonary bypass was established from the inferior vena cava via the femoral vein to the femoral artery. After thoracotomy the bypass was made complete by cannulating the superior vena cava through the right atrium and clamping the inferior vena cava. The left atrium was opened. The prosthesis looked normal from the atrial aspect, but when the ventricular aspect was exposed the prosthesis was seen to be covered with a mass of thrombus which immobilized the disc and which was densely adherent to the stump of one of the papillary muscles (Fig. 2). The prosthesis was replaced with the same type of Starr-Edwards lowprofile disc prosthesis. After bypass the heart action was very poor and in spite of further periods of bypass and other measures the patient died on the table.

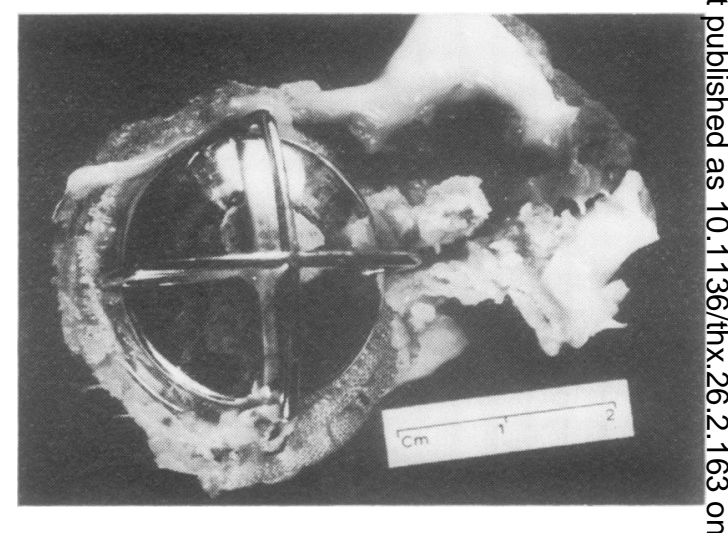

FIG. 2. Starr-Edwards low-profile disc prosthesis (modet $6500)$ removed from case 2 . The papillary muscle on the right of the photograph was firmly adherent to the struts b. thrombus. The thrombus has been pulled off the strut on the right. The groove in which the strut lay can be clearly seengु

Necropsy did not reveal a cause of death, but the lungs showed severe venous congestion.

CASE 3 A 55-year-old woman was seen in March 1968 with a history of rheumatic fever in childhook and angina of effort and breathlessness during the pasg two years. Cardiac catheterization showed the presence of mitral stenosis and incompetence and aortic incom $\overrightarrow{\vec{O}}$ petence. In July 1969 the aortic and mitral valves were replaced with a Starr-Edwards aortic prosthesis (modeb 1200 ) and a Starr-Edwards mitral prosthesis (mode $\vec{E}$ $6500)$ respectively. The operation was uneventful and the patient was started on warfarin 36 hours afte operation. The patient was much improved and remained well until the middle of November 1969 (four months after insertion of the valve) when shes suddenly became breathless. The breathlessness increased and she was admitted to hospital two week later in severe pulmonary oedema. The aortic valire clicks were normal, but no mitral valve clicks coulde be heard. A widespread systolic murmur was presen over the chest, maximal at the apex. An intermitten early diastolic murmur was present at the left sterna edge. A left atrial angiogram showed that the mitra No $^{\circ}$ disc was stuck in the half-open position.

Emergency operation was undertaken after reversa of anticoagulation with plasma prothrombin complexw concentrate. Femoral vein to femoral artery bypass was established before opening the chest through theo previous midsternal incision. Thereafter full bypass్ was achieved by cannulating the superior vena cavas and clamping the inferior vena cava. The mitral disco was found to be fixed in the half-open position byo thrombus within the cage. The mitral valve wasp excised and replaced with a mounted autologous fascio lata valve which had been prepared during the dissection. The aortic prosthesis was inspected and looked normal. For 24 hours after operation the patient's con dition was satisfactory. Thereafter the circulation 
gradually deteriorated with gradual stiffening of the lungs and the development of uraemia. The patient died six days postoperatively.

At necropsy the prosthetic valves looked normal. There was no evidence of myocardial infarction. The lungs were solid and liver-like.

CASE 4 A 44-year-old woman with severe calcific mitral stenosis had her mitral valve replaced with a Starr-Edwards low-profile disc valve (model 6500) in October 1968. The operation was uneventful and the patient made a good recovery. Anticoagulation was maintained with warfarin. At review in December 1969 the patient was well, but in January 1970 (15 months after operation) she was admitted as an emergency, cyanosed, breathless, and with evidence of gross tricuspid incompetence. On auscultation the striking finding was that the valve clicks were audible only intermittently. Loud systolic and diastolic murmurs were heard over the praecordium. Fluoroscopy showed that the metal disc of the valve was tilted in the cage and moved only every third or fourth heart beat.

Urgent replacement of the mitral prosthesis was carried out using the same procedure as has already been described for cases 2 and 3. The prosthesis looked normal when viewed from the atrium, but thrombus on one of the struts had locked the disc in the tilted position (Fig. 3). A previously prepared fascia lata valve was inserted. The patient made a good recovery and is now well.

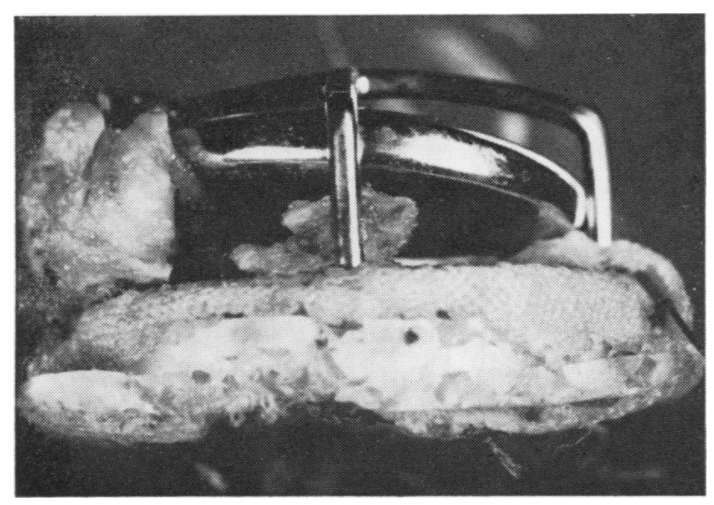

FIG. 3. Starr-Edwards low-profile disc prosthesis (model $6500)$ removed from case 4. Thrombus on the struts has resulted in the disc being tilted in the cage.

\section{DISCUSSION}

Thromboembolism is one of the major problems encountered in the use of artificial heart valves. In some models of Starr-Edwards mitral prostheses the retaining cage is bare metal; there is evidence that this increases the risk of thromboembolism (Davila, Amongero, Sethi, Rincon, Palmer, and
Lautsch, 1966). A reduction in the incidence of thromboembolism followed the covering of the static bare metal with knitted Teflon (Braunwald and Morrow, 1968). In all four cases here described the thrombus formed on the static bare metal part of the prosthesis. The high incidence of this complication in Starr-Edwards low-profile disc prosthesis (model 6500) has led us to abandon the use of this particular model. It is of interest to note that none of our four patients experienced any warning thromboembolic episodes before presenting with acute dysfunction of the prosthesis.

The abrupt onset of dyspnoea was a feature in our cases, but none of the patients died before surgical treatment was available.

Although acute dysfunction of the prosthesis occurring several months after apparently successful mitral valve replacement is uncommon, the diagnosis must be suspected when any patient with a prosthetic valve suddenly deteriorates. The diagnosis is usually easy as disappearance of the valve clicks is readily detected. In the early stages, however, the clicks may be absent only intermittently (Spencer, Trinkle, and Reeves, 1965). Abnormal murmurs may be present but are of little help in making the diagnosis. Marked pulmonary oedema, a feature of the acute dysfunction of the mitral valve, is always present. One of our cases had acute functional tricuspid incompetence which was relieved by changing the faulty mitral prosthesis. In most cases the diagnosis can be made clinically, but if doubt persists fluoroscopy may be helpful in demonstrating abnormal movement of metal balls or discs. Cine-angiography may be required if non-radio-opaque balls or discs have been used.

The only treatment likely to be successful is the urgent replacement of the faulty prosthesis. We made the error in the first patient of assuming that an initial satisfactory response to conservative measures would allow time for reversal of anticoagulation with vitamin K1. Rapid reversal of anticoagulation is desirable and can be achieved by giving plasma prothrombin complex concentrate intravenously (Didisheim, Loeb, Blatrix, and Soulier, 1959).

After our experience with the first case partial cardiopulmonary bypass from femoral vein to femoral artery was subsequently started before thoracotomy. The value of this procedure in supporting the critically ill patient during a difficult exposure of the heart was suggested by Berger and Barsamian (1966). In acute mitral valve failure an additional advantage of partial cardiopulmonary bypass is the relief of pulmonary con- 
gestion obtained by the reduction in venous return to the heart. Cannulation of the superior vena cava after thoracotomy converts the partial bypass to full cardiopulmonary bypass (Samaan and Murali, 1970).

One should not be misled by the normal appearance of the prosthesis when it is first viewed from the left atrium as the thrombus may be present only on the ventricular side of the prosthesis.

Acute dysfunction of the Starr-Edwards mitral prosthesis caused by thrombus is a serious complication with a high mortality. Anticoagulation with warfarin, controlled on an out-patient basis, does not protect against this complication. Survival is likely only if the prosthesis is changed before irreversible cardiac or pulmonary changes have taken place.

I am grateful to Mr. Andrew Logan, Mr. P. R. Walbaum, and Mr. R. J. M. McCormack for permission to publish these case reports. In particular I am grateful to Mr. MoCormack for helpful criticism in the preparation of this paper.

\section{REFERENCES}

Berger, R. L., and Barsamian, E. M. (1966). Iliac or femoral vein-to-artery total cardiopulmonary bypass. Ann. thorac. Surg., 2, 281.

Braunwald, N. S., and Morrow, A. G. (1968). Tissue ingrowth and the rigid heart valve. $J$. thorac. cardiovasc. Surg., 56, 307.

Davila, J. C., Amongero, F., Sethi, R. S., Rincon, N. L., Palmer, T. E., and Lautsch, E. V. (1966). The prevention of thrombosis in artificial cardiac valves. Ann. thorac. Surg., 2, 714.

Didisheim, P., Loeb, J., Blatrix, C., and Soulier, J. P. (1959). Preparation of a human plasma fraction rich in prothrombin, proconvertin, Stuart factor, and PTC and a study of its activity and toxicity in rabbits and man. J. Lab. clin. Med., 53, 322.

Garamella, J. J., Lynch, M. F., Schmidt, W. R., and Jensen, N. K. (1964). Fatal clotting of the Starr-Edwards mitral ball valve nineteen months postoperatively. $J$. thorac. cardiovasc. Surg., 47, 673.

Samaan, H. A., and Murali, R. (1970). Acute tricuspid valve obstruction following the use of tricuspid ball valve prosthesis. Thorax, 25, 334.

Spencer, F. C., Trinkle, J. K., and Reeves, J. T. (1965). Successful replacement of a thrombosed mitral ballvalve prosthesis. J. Amer. med. Ass., 194, 1249.

Starr, A., and Edwards, M. L. (1961). Mitral replacement. Clinical experience with a ball-valve prosthesis. Ann. Surg., 154, 726. 